\title{
OPEN Prognostic efficacy of the RTN1 gene in patients with diffuse large B-cell lymphoma
}

\author{
Mohamad Zamani-Ahmadmahmudi ${ }^{1 凶}$, Seyed Mahdi Nassiri² \& Amir Asadabadi ${ }^{1}$
}

Gene expression profiling has been vastly used to extract the genes that can predict the clinical outcome in patients with diverse cancers, including diffuse large B-cell lymphoma (DLBCL). With the aid of bioinformatics and computational analysis on gene expression data, various prognostic gene signatures for DLBCL have been recently developed. The major drawback of the previous signatures is their inability to correctly predict survival in external data sets. In other words, they are not reproducible in other datasets. Hence, in this study, we sought to determine the gene(s) that can reproducibly and robustly predict survival in patients with DLBCL. Gene expression data were extracted from 7 datasets containing 1636 patients (GSE10846 [ $n=420]$, GSE31312 [ $n=470]$, GSE11318 [ $n=203$ ], GSE32918 [ $n=172$ ], GSE4475 [ $n=123$ ], GSE69051 [ $n=157$ ], and GSE34171 $[n=91]$ ). Genes significantly associated with overall survival were detected using the univariate Cox proportional hazards analysis with a $P$ value $<0.001$ and a false discovery rate (FDR) $<5 \%$. Thereafter, significant genes common between all the datasets were extracted. Additionally, chromosomal aberrations in the corresponding region of the final common gene(s) were evaluated as copy number alterations using the single nucleotide polymorphism (SNP) data of 570 patients with DLBCL (GSE58718 [ $n=242]$, GSE57277 [ $=148]$, and GSE34171 [ $n=180]$ ). Our results indicated that reticulon family gene 1 (RTN1) was the only gene that met our rigorous pipeline criteria and associated with a favorable clinical outcome in all the datasets $(P<0.001, \mathrm{FDR}<5 \%)$. In the multivariate Cox proportional hazards analysis, this gene remained independent of the routine international prognostic index components (i.e., age, stage, lactate dehydrogenase level, Eastern Cooperative Oncology Group [ECOG] performance status, and number of extranodal sites) $(P<0.0001)$. Furthermore, no significant chromosomal aberration was found in the RTN1 genomic region (14q23.1: Start 59,595,976/End $59,870,966)$.

Reticulon family gene 1 (RTN1) (formerly termed "neuroendocrine-specific protein" [NSP]) is a reticulonencoding gene that is associated with the endoplasmic reticulum. Reticulons play critical roles in membrane trafficking or neuroendocrine secretion in neuroendocrine cells. RTN1 encodes 3 variants-namely NSP-A, -B, and $-\mathrm{C}$ - which are attached to the endoplasmic reticulum by means of 2 putative transmembrane domains in the homologous C-terminal region ${ }^{1-4}$.

Previous investigations have introduced $R T N 1$ as a potential diagnostic/therapeutic marker of neurological diseases and cancers ${ }^{2,5-7}$. RTN1 was proposed as a potential marker for carcinomas with neuroendocrine characteristics ${ }^{2}$. It has been shown that RTN1 reduces the anti-apoptotic activity of a protein encoded by BCL2like 1 (BCL2L1) (ie, B-cell lymphoma-extra large [Bcl-xL]). Indeed, RTN1 can change the subcellular localization of the $\mathrm{Bcl}-\mathrm{xL}$ protein from the mitochondria to the endoplasmic reticulum, which disrupts its anti-apoptotic action $^{5}$.

Because of the major shortages of previous prognostic gene signatures developed based on gene expression profiling $^{8-13}$, we sought to find the gene(s) that can reproducibly predict the clinical outcome in patients with diffuse large B-cell lymphoma (DLBCL). Some of the shortcomings of the previous signatures hindering their clinical utility include the infeasibility to reproduce a prognostic signature in external datasets, negligible overlaps between the developed signatures, and large numbers of genes in the developed prognostic genes (180 genes, 90 genes, and 27 genes in signatures developed by Lenz et al. (2008), Alizadeh et al. (2000), and Wright et al. (2003), respectively). In our efforts to find the gene(s) reproducibly associated with survival via bioinformatics and

${ }^{1}$ Department of Clinical Science, Faculty of Veterinary Medicine, Shahid Bahonar University of Kerman, P.O Box 76169133, Kerman, Iran. ${ }^{2}$ Department of Clinical Pathology, Faculty of Veterinary Medicine, University of Tehran, Tehran, Iran. ${ }^{\square}$ email: zamani_2012@uk.ac.ir 


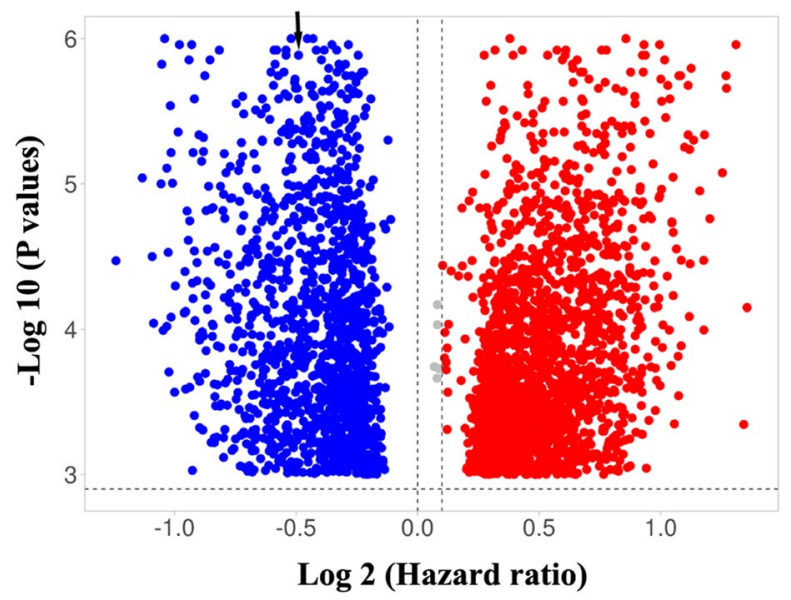

Figure 1. Volcano plot for GSE10846 indicating RTN1 position (arrow) as the most reproducible prognostic gene. Similar plots for other datasets were provided in the Supplementary Figures.

\begin{tabular}{|l|l|l|l|l|l|}
\hline Dataset & HR $^{\mathbf{a}}$ & SE & $\mathbf{9 5 \%}$ CI $^{\mathbf{b}}$ & P value $^{\text {FDR (\%) }}$ \\
\hline GSE10846 & 0.73 & 0.07 & $0.64-0.84$ & $\mathbf{0 . 0 0 0}$ & $\mathbf{0 . 0 3}$ \\
\hline GSE31312 & 0.79 & 0.07 & $0.69-0.90$ & $\mathbf{0 . 0 0 0}$ & $\mathbf{3}$ \\
\hline GSE32918/69051 & 0.78 & 0.07 & $0.68-0.90$ & $\mathbf{0 . 0 0 0}$ & $\mathbf{4}$ \\
\hline GSE4475 & 0.41 & 0.27 & $0.24-0.69$ & $\mathbf{0 . 0 0 0}$ & $\mathbf{4}$ \\
\hline GSE34171 & 0.53 & 0.17 & $0.38-0.73$ & $\mathbf{0 . 0 0 0}$ & $\mathbf{4}$ \\
\hline GSE11318 & 0.69 & 0.10 & $0.57-0.83$ & $\mathbf{0 . 0 0 0}$ & $\mathbf{3}$ \\
\hline
\end{tabular}

Table 1. Statistics of univariate Cox proportional hazard analysis of the RTN1 gene in the various datasets.

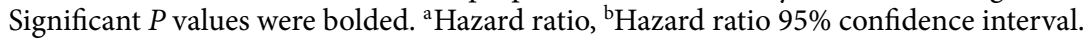

computational approaches, we obtained a surprising result: The RTN1 gene was robustly and reliably associated with a favorable outcome in 1636 patients with DLBCL (including 7 gene expression data sets). Furthermore, the RTN1 gene remained as one of the most powerful independent prognostic factors in comparison with the international prognostic index (IPI) components.

\section{Results}

RTN1 as the most robust and reproducible prognostic gene in all the data sets. First, a univariate Cox proportional hazards analysis was run so as to find genes significantly associated with overall survival in all the datasets. The analysis revealed that 3 genes-namely APOC1, RTN1, and PLAU-fulfilled the criteria and were significantly associated with the clinical outcome at an FDR $<10 \%$ and a $P$ value $<0.001$ (Supplementary Table 1). When the FDR cutoff value was set at 5\%, only RTN1 met our rigorous pipeline criteria and was significantly associated with survival at a $P$ value $<0.001$ in the 1636 patients encompassing all the 7 data sets (Fig. 1 and Supplementary Figures). Except for GSE10846, FDRs on RTN1 were less than 5\% in various datasets (GSE31312: 3\%, GSE32916/69051: 4\%, GSE4475: 4\%, GSE34171: 4\%, and GSE11318: 3\%) $(P s<0.001)$. In GSE10846, FDR was less than $1 \%(P<0.001)$ (Table 1). Meanwhile, this gene showed a consistent positive association with survival in all the data sets (hazard ratio range [HR]: 0.41 to 0.79 ) (Table 1). Overall survival was significantly different between the low-risk and high-risk groups reconstructed based on the median of the $R T N 1$ expression values ( $>$ median value vs. $<$ median value) at a $P$ value $<0.0001$. The rates of overall survival at 5 years in the high-risk and low-risk groups in the different datasets were as follow: GSE31312 (51\% [CI: 45-56\%] vs. 72\% [CI: 66-77\%], $P<0.0001$ ), GSE10846 (48\% [CI: 40-55\%] vs. 67\% [CI: 48-85\%], $P<0.0001)$, GSE32916/69051 (43\% [CI: 38-49\%] vs. $60 \%$ [CI: 50-70\%], $P<0.0001)$, GSE4475 (18\% [CI: 10-26\%] vs. 58\% [CI: 44-73\%], $P<0.0001)$, GSE34171 (60\% [CI: 47-72\%] vs. 87\% [CI: 76-97\%], $P<0.0001)$, and GSE11318 $(36 \%$ [CI: $30-45 \%$ ] vs. $60 \%$ [CI: 45-70\%], $P<0.0001$ ) (Fig. 2). Moreover, $R T N 1$ was differentially expressed between the 2 classes (i.e., long survival vs. short survival) in all the datasets in the SAM analysis $(P<0.001)$.

Additionally, the multivariate Cox proportional hazards analysis indicated that RTN1 remained independent of routine IPI components in both GSE10846 (HR: 0.78 [0.67 to 0.90]) and GSE31312 (HR: 0.77 [0.66 to $0.88])(P s<0.0001)$. Nonetheless, among the IPI parameters, only age remained an independent predictor in both datasets. Some other the IPI factors were only significant in 1 dataset (Table 2). Except for the molecular subtypes, there was no significant correlation between the IPI components and RTN1 expression $(P s>0.05)$. Cases with RTN1 overexpression were more frequent in the GCB-like and type 3 than in the ABC-like subtype $(P s<0.05)$ (Table 3). 

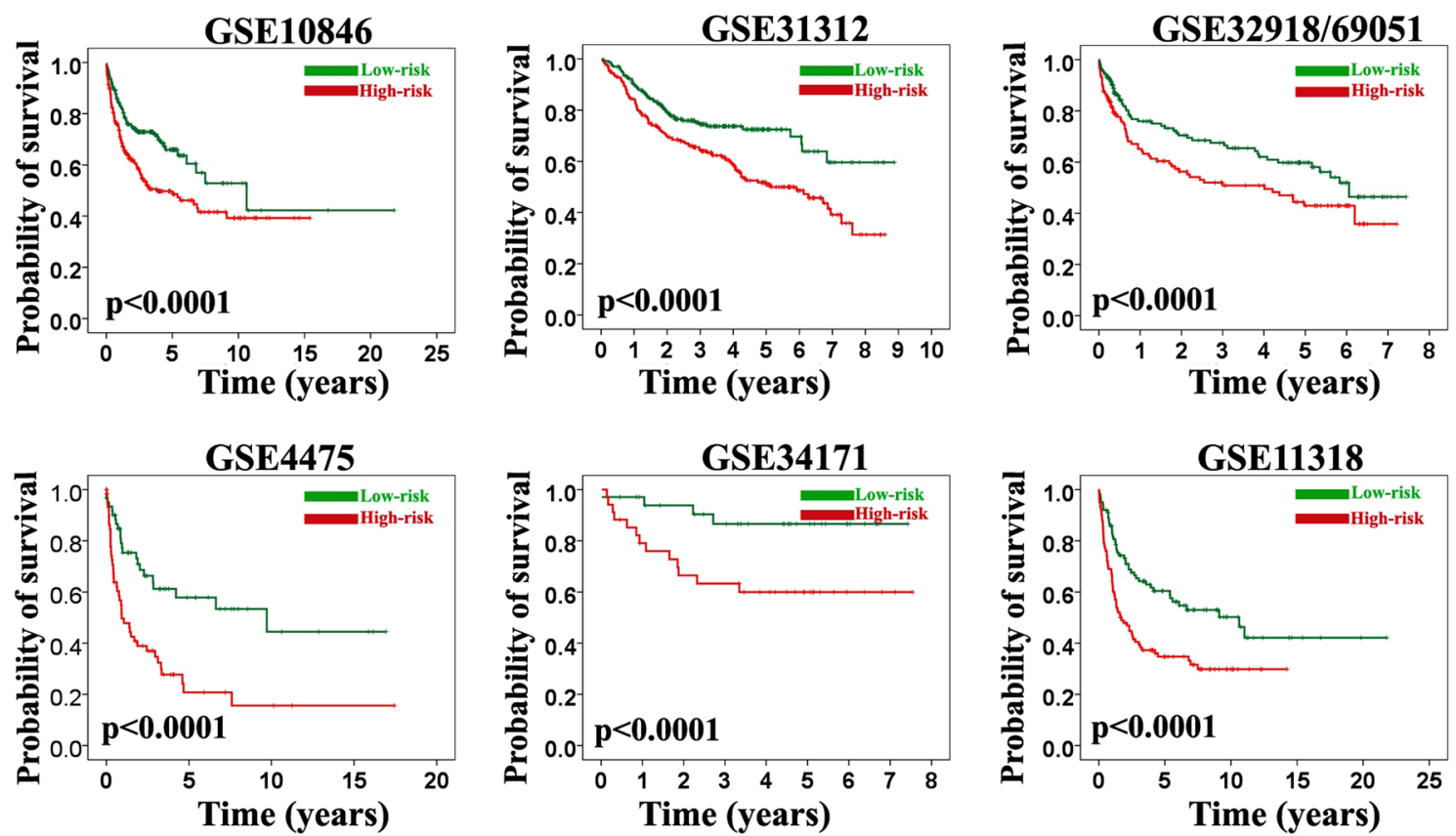

Figure 2. Kaplan-Meier survival analysis of the RTN1 in various gene expression datasets. This gene was found to be significantly associated with the overall survival at a $P$ value $<0.0001$ in all datasets.

\begin{tabular}{|c|c|c|c|c|}
\hline Variable & $H_{R}{ }^{a}$ & SE & $95 \% \mathrm{CI}^{\mathrm{b}}$ & $P$ value \\
\hline \multicolumn{5}{|l|}{ GSE10846 } \\
\hline$R T N 1$ & \begin{tabular}{|l|}
0.78 \\
\end{tabular} & 0.08 & $0.67-0.90$ & 0.000 \\
\hline \multicolumn{5}{|l|}{ Molecular subtype } \\
\hline GCB-like vs. type 3 & 0.98 & 0.30 & $0.55-1.76$ & 0.96 \\
\hline ABC-like vs. type 3 & 1.36 & 0.29 & $0.78-2.39$ & 0.28 \\
\hline Age ( $\geq 60$ vs. $<60$ years) & 1.88 & 0.18 & $1.32-2.68$ & 0.000 \\
\hline Sex (male vs. female) & 1.23 & 0.17 & $0.89-1.72$ & 0.22 \\
\hline Stage (III/IV vs. I/II) & 1.81 & 0.20 & $1.23-2.67$ & 0.000 \\
\hline $\operatorname{NES}^{\mathrm{c}}(\geq 2$ vs. $<2)$ & 1.62 & 0.19 & $1.12-2.33$ & 0.01 \\
\hline ECOG $^{\mathrm{d}}(\geq 2$ vs. $<2)$ & 1.50 & 0.20 & $1.03-2.20$ & 0.04 \\
\hline $\mathrm{LDH}^{\mathrm{e}}$ & 1.58 & 0.20 & $1.07-2.35$ & 0.02 \\
\hline \multicolumn{5}{|l|}{ GSE31312 } \\
\hline RTN1 & 0.77 & 0.07 & $0.66-0.88$ & 0.000 \\
\hline \multicolumn{5}{|l|}{ Molecular subtype } \\
\hline GCB-like vs. type 3 & 0.75 & 0.30 & $0.41-1.35$ & 0.33 \\
\hline ABC-like vs. type 3 & 1.63 & 0.28 & $0.95-2.81$ & 0.08 \\
\hline Age ( $\geq 60$ vs. $<60$ years) & 2.23 & 0.20 & $1.51-3.31$ & 0.000 \\
\hline Sex (male vs. female) & 1.04 & 0.18 & $0.73-1.50$ & 0.82 \\
\hline Stage (III/IV vs. I/II) & 1.30 & 0.20 & $0.88-1.92$ & 0.20 \\
\hline NES ( $\geq 2$ vs. $<2$ ) & 1.16 & 0.34 & $0.59-2.27$ & 0.67 \\
\hline ECOG $(\geq 2$ vs. $<2)$ & 2.23 & 0.20 & $1.52-3.27$ & 0.000 \\
\hline LDH & 1.12 & 0.03 & \begin{tabular}{|l|}
$1.06-1.18$ \\
\end{tabular} & \begin{tabular}{|l|}
0.000 \\
\end{tabular} \\
\hline
\end{tabular}

Table 2. Multivariate analysis of the RTN1 and common prognostic variables in DLBCL (the IPI components). The RTN1 gene was remained as a one of the most powerful independent prognostic factor. Significant $P$ values were bolded. ${ }^{a}$ Hazard ratio, b Hazard ratio $95 \%$ confidence interval, c No. of extranodal sites, dECOG performance status, ${ }^{\mathbf{e}}$ Lactate dehydrogenase. 


\begin{tabular}{|l|l|l|}
\hline Component & GSE31312 & GSE10846 \\
\hline Molecular subtype & $\mathbf{0 . 0 4 5}$ & $\mathbf{0 . 0 3 1}$ \\
\hline Age & 0.351 & 0.324 \\
\hline Sex & 0.113 & 0.660 \\
\hline Stage & 0.579 & 0.927 \\
\hline NES & 0.657 & 0.870 \\
\hline ECOG & 0.819 & 0.643 \\
\hline LDH & 0.141 & 0.050 \\
\hline
\end{tabular}

Table 3. Correlation between the IPI components and RTN1 expression. Significant $P$ value was bolded.

Expression of RTN1 in the molecular subtypes of DLBCL. The expression of RTN1 was compared between the different molecular subtypes of DLBCL (i.e., ABC-like, GCB-like, and type 3) using the one-way ANOVA test. The examination indicated that the expression of RTN1 was significantly higher in the subtype with the better overall survival (i.e., GCB-like) than in the subtype with the inferior survival (i.e., ABC-like) $(P s<0.05)$ in both GSE10846 and GSE31312. We also checked whether overall survival was significantly different between the groups based on RTN1 in the different molecular subtypes of DLBCL. Our analysis revealed that overall survival was significantly different between the 2 risk groups in the GCB-like subtype $(P s<0.05)$, whereas there was no significant association between the 2 risk groups in the other subtypes (i.e., ABC-like and type 3) (Fig. 3).

Correlation between RTN1, BCL2L1, and MYC expressions. Tagamei et al. (2000) indicated that RTN1 only changes the subcellular localization of Bcl-xL from mitochondria to the endoplasmic reticulum and does not alter the expression level of the corresponding gene (i.e., BCL2L1). Our analysis revealed no significant and consistent correlations between the RTN1 probe-sets $(\mathrm{n}=2)$ and the $B C L 2 L 1$ probe-sets $(\mathrm{n}=4)$, where some inconsistent (a mix of positive and negative results) and poor correlation coefficients $(r<0.59)$ were obtained in the different analyses (Fig. 4 and Supplementary Table 2). Nevertheless, an elevation in RTN1 expression did not suppress BCL2L1 expression. There was a good and significant correlation between the 2 RTN1 probe-sets $(r=0.82, P<0.01)$ (Fig. 5 and Supplementary Table 2). On the other hand, these were a significant stable negative correlation between RTN1 and MYC expression in analyzed datasets $(P s<0.05)$ although the correlation coefficients were not strong enough $(r<0.59)$ (Supplementary Table 3).

Association between RTN1 and apoptosis or cell trafficking pathways. Our GSEA analysis revealed that the genes involved in the apoptosis pathway (i.e. HALLMARK_APOTOSIS) as well as one of the cell trafficking gene-sets (i.e. GOCC_ENDOCYTIC_VESICLE_MEMBRANE) were clearly enriched in the low-risk groups (higher RTN1 expression) compared with the high-risk group (lower RTN1 expression) in all datasets $(P s<0.05)$. Furthermore, GOBP_EXOCYTIC_PROCESS pathway was also significantly enriched in the low-risk group in all datasets except GSE10846 and GSE31312 (Ps<0.05) (Fig. 6 and Supplementary Table 4).

RTN1 at the genome level. Possible CNAs of the RTN1 gene (14q23.1: Start 59,595,976/End 59,870,966) were checked through an analysis on the SNP data of 570 patients with DLBCL. The upstream and downstream regions that might partly include the RTN1 region were also explored in order to detect possible aberrations. The analysis indicated no significant chromosomal aberrations in the region of the RTN1 gene (14q23.1: Start $59,595,976 /$ End 59,870,966). Nonetheless rare chromosomal gains $(\mathrm{CN}=3$, amplification of 59,526,724$60,901,663$ segment $)$ and losses $(\mathrm{CN}=1$, loss of 59,580,209-60,388,423 segment) were detected in 2 samples of GSE58718 and GSE34171, correspondingly, where these abnormalities were not recurrent (frequency $<1 \%$ ) (Supplementary Table 5). No chromosomal instability was found in the region of interest in GSE57277.

\section{Discussion}

Several studies have proposed various prognostic signatures comprising different numbers of genes via gene expression analysis ${ }^{8-14}$. Indeed, there is a minimum of overlap between these signatures and in many situations, there is no common gene in the suggested signatures. In addition, we found no genes of the previous signatures that were reproducibly associated with the clinical outcome in our data sets. Another disadvantage of these signatures is their large number of genes. Indubitably, the use of such large signatures is impractical in routine clinical practice ${ }^{15,16}$.

In the current study, we assessed the prognostic efficacy of RTN1 in several large cohorts of patients with DLBCL. This gene was consistently associated with a favorable outcome in all the datasets, comprised of 1636 patients with DLBCL. The association between the RTN1 gene expression and a favorable clinical outcome in all the datasets was significant at an $\mathrm{FDR}<5 \%$, which means that the probability of a false positive was extremely low. Furthermore, it remained as a one of the most powerful independent prognostic factors in comparison with the IPI components. Although RTN1 was not the most powerful gene associated with overall survival, it was the only gene that reproducibly predicted a favorable clinical outcome. This gene was previously reported as a member of the stromal-1 signature in a 108-gene model developed by Lenz et al. (2008). Additionally, the upregulation of 



Figure 3. Comparison of expression of our predictor gene (RTN1) in three molecular subtypes of DLBCL (i.e. ABC-like, GCB-like, and type 3). Left and right panels indicate GSE10846 and GSE31312 datasets, respectively. The survival time in GCB-like was significantly different between two risk groups $(P s<0.05)$.

RTN1 in CXCR4- DLBCL versus CXCR4 + DLBCL was indicated, where CXCR4- and CXCR4 + subtypes were associated with better and poorer overall survival, respectively ${ }^{17}$.

Various roles of RTN1 in the biology of cancers have been previously investigated. As was previously described in the introduction, RTN1 induces its antitumor activity through interaction with $\mathrm{Bcl}-\mathrm{xL}$ on the endoplasmic reticulum and reduces its anti-apoptotic activity ${ }^{5}$. A previous investigation revealed that a member of the RTN family (ie, RTN-1C) sensitizes neuroepithelioma cells to fenretinide-induced apoptosis through interaction with glucosylceramide synthase ${ }^{18}$. Moreover, $R T N 1$-encoded proteins have been proposed as a category criterion for human lung cancer ${ }^{19}$. Previous research has also demonstrated that NSP-reticulon expression is restricted to lung carcinoma cells with a neuroendocrine phenotype ${ }^{6,7,19}$. Another RTN1 paralog (i.e., RTN3) has a similar antitumor activity through the enrichment of TRAIL-mediated apoptosis via the downregulation of c-FLIP and the upregulation of death receptor $5^{20}$.

In light of the results of the present study, RTN1 can be considered as a potential prognostic gene capable of predicting survival in patients with DLBCL. Further studies are to be conducted to explore the prognostic efficacy of RTN1 in depth. In this way, the prognostic efficacy of this gene should be well again compared with the regular prognostic parameters. This gene should also be experimentally validated in large cohorts of the patients with DLBCL. Because of some major limitations, we could not collect enough homogenous DLBCLs 

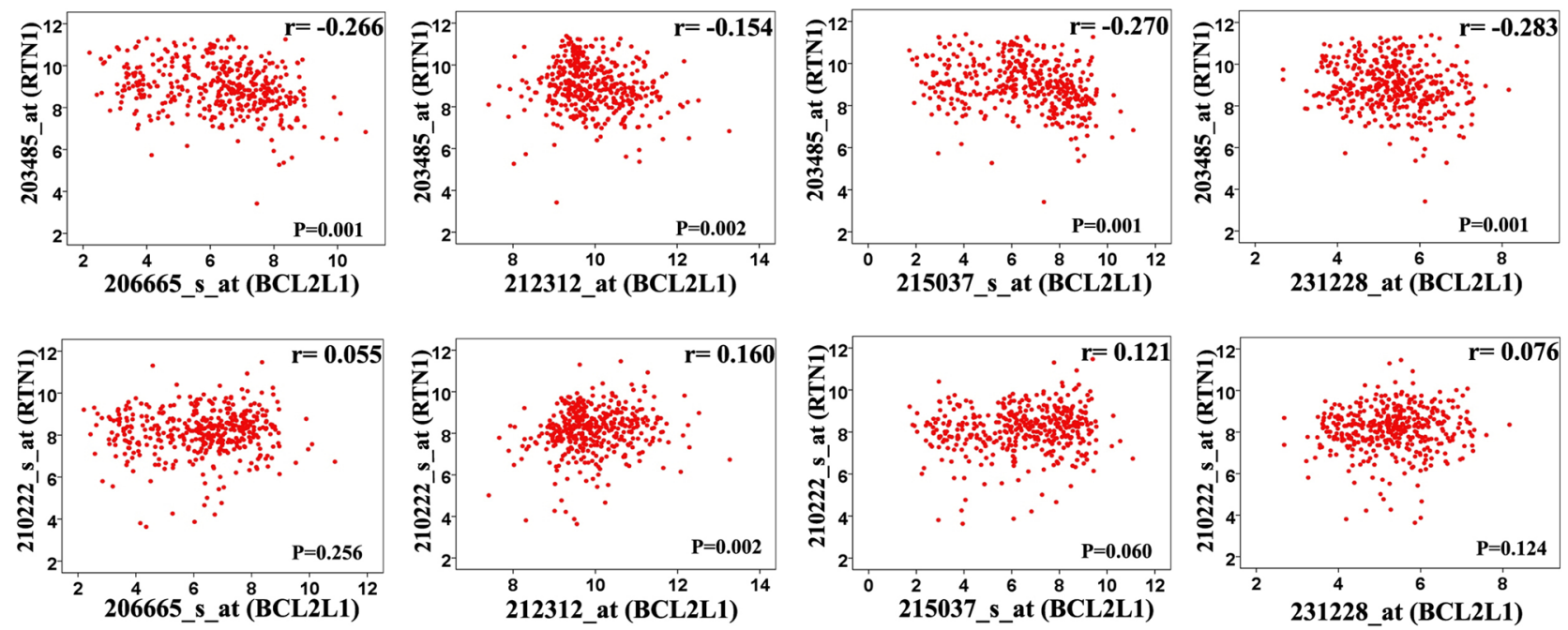

Figure 4. Representing scatterplot depicting the correlation between various BCL2L1 probe-sets (x-axis) and RTN1 probe-sets (y-axis) in GSE10846. The correlations between pairs of probes were poor and inconsistent (negative and positive correlations). Correlation analysis for other datasets was presented in Supplementary Table 2.

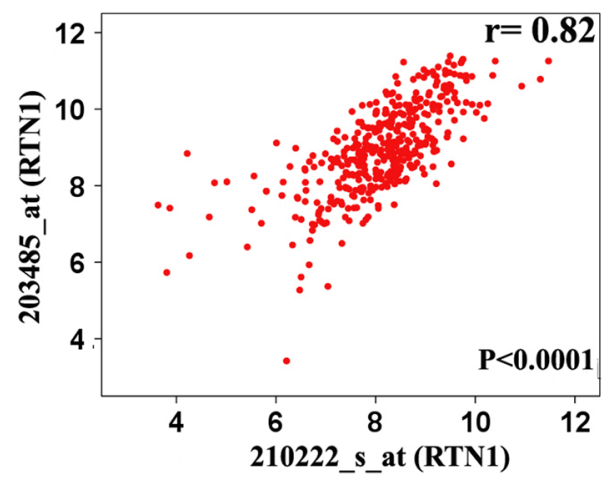

Figure 5. Representative scatterplot depicting the correlation between two RTN1 probe-sets (203485_at and 210222_s_at). Correlation coefficient between two probe-sets was statistically significant $(\mathrm{r}=0.82)(P<0.05)$.

with the confirmed survival time for subsequent experimental procedure. Indeed, survival time as the most important clinical metadata was not available for many patients in our clinical settings. Because gene expression profiling using the microarray technology is carried out with various molecular chips, heterogeneous expression patterns may be resulted. In addition, high-output methods are more time-consuming and cost-effective than simpler ones. It is recommended that the prognostic efficacy of RTN1 is evaluated in more annotated cohorts with DLBCL using subtle techniques such as PCR, quantitative real-time PCR, immunohistochemistry (IHC) to validate the current findings.

\section{Materials and methods}

Datasets. The Gene Expression Omnibus (GEO) (https://www.ncbi.nlm.nih.gov/geo/) database was searched to find the gene expression profiling datasets of patients with DLBCL. Only datasets containing clinical metadata (especially overall survival) (7 datasets) were retained, and the rest were excluded. Additionally, every effort was made to select expression datasets from all types of microarray chips such as Affymetrix and Illumina, if possible. The datasets were downloaded in SOFT file format and were subsequently transformed logarithmically using tools provided in geWorkbench 2.5.1 package $^{21}$, if necessary. More details on the clinical characteristics of the studied datasets are provided in Table 4. The datasets included GSE10846 $(n=420)$, GSE31312 $(n=470)$, GSE32918 $(n=172)$, GSE69051 $(n=157)$, GSE4475 $(n=123)$, GSE11318 $(n=203)$, and GSE34171 $(n=91)$. The analyzed datasets including GSE10846, GSE4475, GSE11318, and GSE69051 contained patients treated with both R-CHOP and CHOP regimens. Furthermore, GSE31312, GSE32918 and GSE34171 contained patients treated with R-CHOP regimen (Table 4). Since GSE32918 and GSE69051 have originated from a similar research study ${ }^{22}$ and have some common samples, they were merged as a single data set and termed "GSE32918/69051". The number of samples for these datasets was determined after corrections were made based on the common samples (172 samples for GSE32918 and 157 samples for GSE69051). In addition, the genetic aberrations of the 
Enrichment plot: HALLMARK_APOPTOSIS

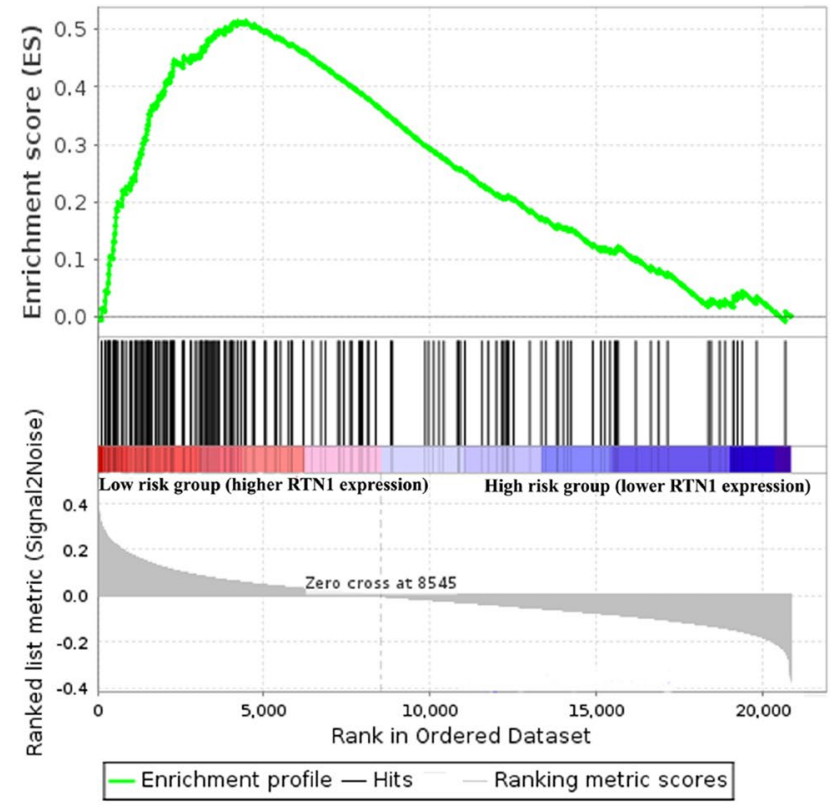

Enrichment plot: GOCC_ENDOCYTIC_VESICLE_MEMBRANE

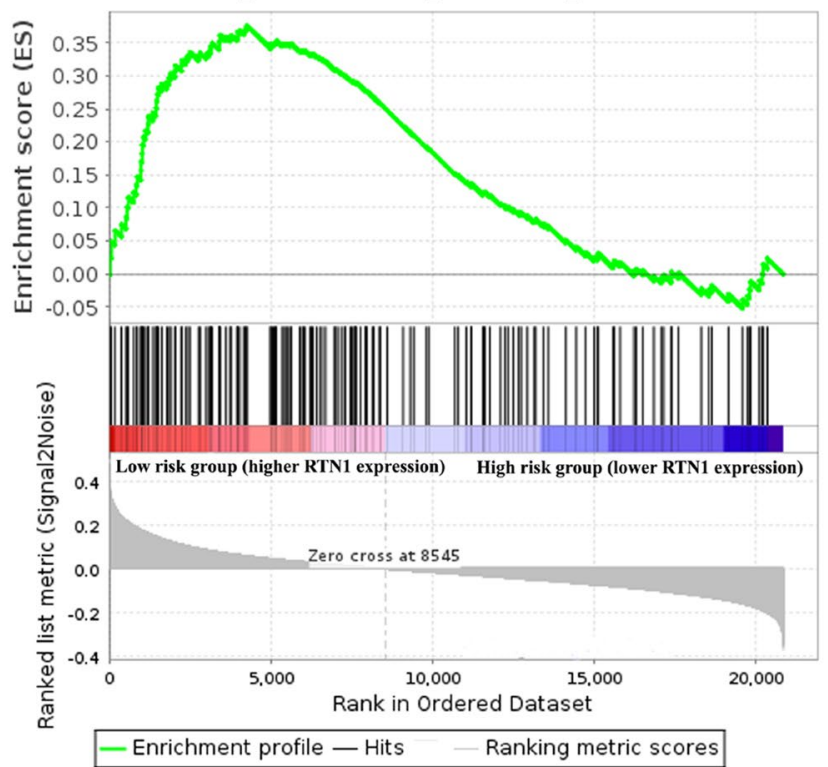

Figure 6. Representative gene set enrichment analysis (GSEA) indicating the enrichment of HALLMARK APOTOSIS and GOCC_ENDOCYTIC_VESICLE_MEMBRANE gene sets in low-risk group (higher RTN1 expression) in GSE10846 (Ps < 0.05). Statistics of GSEA for other datasets were provided in the Supplementary Table 4.

\begin{tabular}{|l|l|l|l|l|l|}
\hline Dataset & Number of patients & Chip manufacturer & Platform & Treatment & Usage in our study \\
\hline GSE10846 & 420 & Affymetrix & GPL570 & R-CHOP/ CHOP & Survival analysis \\
\hline GSE31312 & 470 & Affymetrix & GPL570 & R-CHOP & Survival analysis \\
\hline GSE32918 & 172 & Illumina & GPL8432 & R-CHOP & Survival analysis \\
\hline GSE69051 & 157 & Illumina & GPL14951 & R-CHOP/CHOP & Survival analysis \\
\hline GSE4475 & 123 & Affymetrix & GPL96 & R-CHOP/CHOP & Survival analysis \\
\hline GSE11318 & 203 & Affymetrix & GPL570 & R-CHOP/CHOP & Survival analysis \\
\hline GSE34171 & 91 & Affymetrix & GPL570 & R-CHOP & Survival analysis \\
\hline GSE58718 & 242 & Illumina & GPL6986 & NR ${ }^{a}$ & Chromosomal aberration \\
\hline GSE57277 & 148 & Affymetrix & GPL3720 & NR & Chromosomal aberration \\
\hline GSE34171 & 180 & Affymetrix & GPL6801 & R-CHOP & Chromosomal aberration \\
\hline
\end{tabular}

Table 4. Clinical characteristics of the microarray datasets used in our study. ${ }^{\text {a }}$ Not recorded.

desired gene(s) at the genome level were evaluated by employing 3 single nucleotide polymorphism (SNP) array data sets-namely GSE58718 $(\mathrm{n}=242)$, GSE57277 $(\mathrm{n}=148)$, and GSE34171 $(\mathrm{n}=180)$. GSE34171 contains both gene expression and SNP data (Table 4).

Identification of the common gene(s) associated with survival in the gene expression data sets. The association between gene expression and overall survival was examined using the univariate Cox proportional hazards analysis as previously described ${ }^{23,24}$. In this analysis, the association between a group of covariates (genes) and the response variable (overall survival) was evaluated. The univariate Cox analysis was performed using BRB-Array tools, developed by Richard Simon and the BRB-ArrayTools Development Team. In this analysis, the findings were strengthened by employing rigorous pipeline criteria and retaining only genes with a $P$ value $<0.001$ and a false discovery rate (FDR) $<5 \%$. Subsequently, the common gene(s) significantly associated with overall survival between all the data sets was/were extracted. For this purpose, only common gene(s) with consistent associations were selected, while genes with inconsistent associations (negatively associated with overall survival in a dataset and positively associated with overall survival in another) were excluded. Moreover, the patients were categorized into 2 risk groups (high-risk vs. low-risk) based on the median of the selected common gene expression values ( $>$ median value vs. $<$ median value), and overall survival was compared between the groups using the Kaplan-Meier analysis and log-rank test at a $P$ value $<0.01$. The KaplanMeier analysis and the log-rank test were performed in SPSS 16.0 package (Chicago, USA). Since RTN1 was the 
only gene that fulfilled the criteria and was selected as the final gene, the subsequent analyses were exclusively performed on this gene. The prognostic efficacy of RTN1 in various datasets was visualized through depicting a volcano plot $^{25}$, where the hazard ratios were plotted against the logarithmic values of $P$ score obtained from Cox analysis.

As a confirmatory step, we checked whether RTN1 was differentially expressed between the 2 predefined survival classes using the significance analysis of microarray (SAM) analysis. In this analysis, 2 classes (long survival $[\geq 5 \mathrm{y}]$ vs. short survival $[<5 \mathrm{y}]$ ) were created and, thereafter, the genes that were differentially expressed were detected. The SAM analysis was performed using the method added in BRB-Array tools. In this analysis, the FDR and the number of permutations were set at $5 \%$ and 1000 , respectively.

Prognostic efficacy of the RTN1 gene in a multivariate model. The prognostic efficacy of RTN1 was also evaluated in a multivariate Cox proportional-hazards regression analysis, where the RTN1 gene expression and all the individual components of the international prognostic index (IPI) (ie, age, stage, lactate dehydrogenase level, Eastern Cooperative Oncology Group [ECOG] performance status, and number of extranodal sites) ${ }^{26}$ were entered as covariate variables. Additionally, the molecular subtype (ie, ABC-like, GCB-like, and type 3) and sex were incorporated as another 2 variables into the model. 3 . The IPI components in two datasets (i.e. GSE31312 and GSE10846) were treated (adjusted) as ordinal variables. Hence, two or three codes were assigned to each variable $(0$ and 1 or $0,1,2)$. Therefore, the components were considered as follow: molecular subtypes (GCB-like, ABC-like, type 3 ); age ( $\geq 60$ and $<60$ years); sex (male and female); stage (III/IV and I/II); NES ( $\geq 2$ and $<2$ ); ECOG4 ( $\geq 2$ vs. $<2)$; and LDH (0 vs. 1$)$. The multivariate analysis was performed on the datasets with the available clinical IPI data (i.e., GSE10846 and GSE31312). This analysis was carried out using Survival package (http://cran.r-project.org/package=survival) and SPSS 16.0 package (Chicago, USA).

Furthermore, the correlation between RTN1 expression and the IPI components was evaluated using the Pearson chi-squared test. In this analysis, patients were divided into two overexpression and none-overexpression groups based on RTN1 expression.

Prognostic efficacy of the RTN1 gene in molecular subtypes of DLBCL. We also checked the prognostic worth of RTN1 in molecular subtypes of DLBCL (i.e., ABC-like, GCB-like, and type 3) using similar strategy described above. In brief, in each subtypes two risk groups constituted based on median of the RTN1 expression values ( $>$ median value vs. $<$ median value) and then overall survival was compared between the groups using the Kaplan-Meier analysis and log-rank test at a $P$ value $<0.01$.

Correlation between RTN1, BCL2L1, and MYC expressions. One of the main targets of RTN1 is $B C L 2 L 1$ via the inhibition of its anti-apoptotic activity ${ }^{5}$. Moreover, BCL2L1 is a member of BCL-2 protein families deregulated in lymphoma tumors, especially DLBCL ${ }^{27-29}$. Accordingly, the associations between 2 probe-sets of RTN1 (i.e., 203485_at and 210222_s_at) and 4 probe-sets of BCL2L1 (i.e., 206665_s_at, 212312_at, 215037_s_ at, and 231228_at) were evaluated using correlation analysis. We also analyzed correlation between one of the most important proto-oncogenes involved in DLBCL (i.e. $c-M Y C$ ) and RTN1. As well, the association analysis between C-MYC (202431_s_at) and RTN1 probe-sets was carried out. The correlations were graded based on the classification proposed by Papasouliotis et al. ${ }^{30}$ (i.e., $r=0.93$ to 0.100 as excellent, $r=0.80$ to 0.92 as good, $r=0.59$ to 0.79 as fair, and $r<0.59$ as poor correlations). The correlation analysis was performed using SPSS 16.0 package (Chicago, USA) in all the datasets, and a $P$ value $<0.05$ was considered significant.

Association between RTN1 and apoptosis as well as cell trafficking pathways. The associations between RTN1 and genes involved in apoptosis and cell trafficking pathways were evaluated using the Gene-Set Enrichment Analysis (GSEA) ${ }^{31}$. Apoptosis pathway (gene-set) (i.e. HALLMARK_APOTOSIS) and two cell trafficking gene-sets (i.e. GOCC_ENDOCYTIC_VESICLE_MEMBRANE, GOBP_EXOCYTIC_PROCESS) were retrieved from the Molecular Signatures Database (MSigDB) (http://software.broadinstitute.org/gsea/index. jsp). Then, the expression patterns of these gene-sets were compared between the low-risk and high-risk groups reconstructed based on the median of the RTN1 expression values ( $>$ median value vs. $<$ median value) in different datasets. These analyzes were run on 1000 permutations.

Evaluation of RTN1 at the genome level in the DLBCL samples. For the assessment of the chromosomal aberrations of the RTN1 gene, 3 datasets-namely GSE58718 $(\mathrm{n}=242)$, GSE57277 $(\mathrm{n}=148)$, and GSE34171 ( $\mathrm{n}=180$ ) - were used to extract copy number variations (CNVs) from the SNP data. GSE58718 was generated based on Illumina HumanCNV370-Duov1 DNA Analysis BeadChip, while GSE57277 and GSE34171 were generated using Affymetrix Mapping $250 \mathrm{~K}$ SNP Arrays. In brief, PennCNV package ${ }^{32}$ was used to call and analyze the CNV data. For the Illumina datasets, signal intensity data in the form of log R ratios (LRRs) and B allele frequencies (BAFs) were directly generated from the downloaded raw file. For the Affymetrix datasets, LRRS and BAFs were calculated by processing raw intensity (.CEL) files in Affymetrix Power Tools (https://www. affymetrix.com/support/developer/powertools/changelog/index.html), followed by PennCNV-Affy package. Finally, these LRRS and BAFs were used to generate CNV calls. CNVs with lengths $<1 \mathrm{~kb}$, confidence scores $<10$, or containing $<5$ SNPs were discarded. A CNV was considered to be a recurring acquired copy number alteration (rCNA) if it occurred in more than $2.5 \%$ of the patients and was not reported in the Database of Genomic Variants, build 36 (hg18) (DGV, http://projects.tcag.ca/variation/) ${ }^{33}$. The location of RTN1 was explored on chromosome 14 (14q23.1: Start 59,595,976/End 59,870,966) for chromosomal aberrations. 
Ethical standards. Our study was performed using datasets deposited in GEO database. Hence, no ethical approval was required.

\section{Data availability}

The datasets in the manuscript were deposited in GEO database (http://www.ncbi.nlm.nih.gov/geo/) with the accession number GSE10846, GSE31312, GSE32918, GSE69051, GSE4475, GSE11318, GSE34171, GSE58718, GSE57277, and GSE34171. Other supporting data are included as supplementary files.

Received: 2 August 2021; Accepted: 11 October 2021

Published online: 26 October 2021

\section{References}

1. Roebroek, A. J., Contreras, B., Pauli, I. G. \& Van de Ven, W. J. cDNA cloning, genomic organization, and expression of the human RTN2 gene, a member of a gene family encoding reticulons. Genomics 51, 98-106. https://doi.org/10.1006/geno.1997.5175 (1998).

2. Roebroek, A. J. et al. Cloning and expression of alternative transcripts of a novel neuroendocrine-specific gene and identification of its 135-kDa translational product. J. Biol. Chem. 268, 13439-13447 (1993).

3. Senden, N. H. et al. Neuroendocrine-specific protein C (NSP-C): Subcellular localization and differential expression in relation to NSP-A. Eur. J. Cell Biol. 69, 197-213 (1996).

4. GrandPré, T., Nakamura, F., Vartanian, T. \& Strittmatter, S. M. Identification of the Nogo inhibitor of axon regeneration as a reticulon protein. Nature 403, 439-444. https://doi.org/10.1038/35000226 (2000).

5. Tagami, S., Eguchi, Y., Kinoshita, M., Takeda, M. \& Tsujimoto, Y. A novel protein, RTN-XS, interacts with both Bcl-XL and Bcl-2 on endoplasmic reticulum and reduces their anti-apoptotic activity. Oncogene 19, 5736-5746. https://doi.org/10.1038/sj.onc.12039 48 (2000).

6. Senden, N. et al. Neuroendocrine-specific protein (NSP)-reticulons as independent markers for non-small cell lung cancer with neuroendocrine differentiation. An in vitro histochemical study. Histochem. Cell Biol. 108, 155-165 (1997).

7. Senden, N. H. et al. A comparison of NSP-reticulons with conventional neuroendocrine markers in immunophenotyping of lung cancers. J. Pathol. 182, 13-21. https://doi.org/10.1002/(sici)1096-9896(199705)182:1\%3c13::aid-path804\%3e3.0.co;2-z (1997).

8. Alizadeh, A. A. et al. Distinct types of diffuse large B-cell lymphoma identified by gene expression profiling. Nature 403, 503-511. https://doi.org/10.1038/35000501 (2000).

9. Lossos, I. S. et al. Prediction of survival in diffuse large-B-cell lymphoma based on the expression of six genes. N. Engl. J. Med. 350, 1828-1837. https://doi.org/10.1056/NEJMoa032520 (2004).

10. Rosenwald, A. et al. The use of molecular profiling to predict survival after chemotherapy for diffuse large-B-cell lymphoma. $N$. Engl. J. Med. 346, 1937-1947. https://doi.org/10.1056/NEJMoa012914 (2002).

11. Wright, G. et al. A gene expression-based method to diagnose clinically distinct subgroups of diffuse large B cell lymphoma. Proc. Natl. Acad. Sci. U. S. A. 100, 9991-9996. https://doi.org/10.1073/pnas.1732008100 (2003).

12. Lenz, G. et al. Stromal gene signatures in large-B-cell lymphomas. N. Engl. J. Med. 359, 2313-2323. https://doi.org/10.1056/NEJMo a0802885 (2008).

13. Shipp, M. A. et al. Diffuse large B-cell lymphoma outcome prediction by gene-expression profiling and supervised machine learning. Nat. Med. 8, 68-74. https://doi.org/10.1038/nm0102-68 (2002).

14. Zamani-Ahmadmahmudi, M. \& Nassiri, S. M. Development of a reproducible prognostic gene signature to predict the clinical outcome in patients with diffuse large B-cell lymphoma. Sci. Rep. https://doi.org/10.1038/s41598-019-48721-0 (2019).

15. Alizadeh, A. A. et al. Prediction of survival in diffuse large B-cell lymphoma based on the expression of 2 genes reflecting tumor and microenvironment. Blood 118, 1350-1358. https://doi.org/10.1182/blood-2011-03-345272 (2011).

16. Haibe-Kains, B., Desmedt, C., Sotiriou, C. \& Bontempi, G. A comparative study of survival models for breast cancer prognostication based on microarray data: Does a single gene beat them all?. Bioinformatics (Oxford, England) 24, 2200-2208. https://doi.org/ 10.1093/bioinformatics/btn374 (2008).

17. Chen, J. et al. Dysregulated CXCR4 expression promotes lymphoma cell survival and independently predicts disease progression in germinal center B-cell-like diffuse large B-cell lymphoma. Oncotarget 6, 5597-5614. https://doi.org/10.18632/oncotarget.3343 (2015).

18. Di Sano, F. et al. Glucosylceramide synthase and its functional interaction with RTN-1C regulate chemotherapeutic-induced apoptosis in neuroepithelioma cells. Can. Res. 63, 3860-3865 (2003).

19. van de Velde, H. J. et al. NSP-encoded reticulons are neuroendocrine markers of a novel category in human lung cancer diagnosis. Can. Res. 54, 4769-4776 (1994).

20. Lee, J. T., Lee, T.-J., Kim, C.-H., Kim, N.-S. \& Kwon, T. K. Over-expression of reticulon 3 (RTN3) enhances TRAIL-mediated apoptosis via up-regulation of death receptor 5 (DR5) and down-regulation of c-FLIP. Cancer Lett. 279, 185-192. https://doi.org/ 10.1016/j.canlet.2009.01.035 (2009).

21. Floratos, A., Smith, K., Ji, Z., Watkinson, J. \& Califano, A. geWorkbench: An open source platform for integrative genomics. Bioinformatics 26, 1779-1780. https://doi.org/10.1093/bioinformatics/btq282 (2010).

22. Barrans, S. L. et al. Whole genome expression profiling based on paraffin embedded tissue can be used to classify diffuse large B-cell lymphoma and predict clinical outcome. Br. J. Haematol. 159, 441-453. https://doi.org/10.1111/bjh.12045 (2012).

23. Zamani-Ahmadmahmudi, M., Nassiri, S. M. \& Soltaninezhad, F. Development of an RNA sequencing-based prognostic gene signature in multiple myeloma. Br. J. Haematol. 192, 310-321. https://doi.org/10.1111/bjh.16744 (2021).

24. Zamani-Ahmadmahmudi, M., Dabiri, S. \& Nadimi, N. Identification of pathway-based prognostic gene signatures in patients with multiple myeloma. Transl. Res.: J. Lab. Clin. Med. 185, 47-57. https://doi.org/10.1016/j.trsl.2017.05.001 (2017).

25. Goedhart, J. \& Luijsterburg, M. S. VolcaNoseR is a web app for creating, exploring, labeling and sharing volcano plots. Sci. Rep. 10, 20560. https://doi.org/10.1038/s41598-020-76603-3 (2020).

26. Sehn, L. H. \& Gascoyne, R. D. Diffuse large B-cell lymphoma: Optimizing outcome in the context of clinical and biologic heterogeneity. Blood 125, 22-32. https://doi.org/10.1182/blood-2014-05-577189 (2015).

27. Tsuyama, N. et al. BCL2 expression in DLBCL: Reappraisal of immunohistochemistry with new criteria for therapeutic biomarker evaluation. Blood 130, 489-500. https://doi.org/10.1182/blood-2016-12-759621 (2017).

28. Iqbal, J. et al. BCL2 expression is a prognostic marker for the activated B-cell-like type of diffuse large B-cell lymphoma. J. Clin. Oncol.: Off. J. Am. Soc. Clin. Oncol. 24, 961-968. https://doi.org/10.1200/jco.2005.03.4264 (2006).

29. Iqbal, J. et al. BCL2 translocation defines a unique tumor subset within the germinal center B-cell-like diffuse large B-cell lymphoma. Am. J. Pathol. 165, 159-166 (2004).

30. Papasouliotis, K. et al. Comparison of white blood cell differential percentages determined by the in-house LaserCyte hematology analyzer and a manual method. Vet. Clin. Pathol. 35, 295-302 (2006).

31. Subramanian, A. et al. Gene set enrichment analysis: A knowledge-based approach for interpreting genome-wide expression profiles. Proc. Natl. Acad. Sci. U. S. A. 102, 15545-15550. https://doi.org/10.1073/pnas.0506580102 (2005). 
32. Wang, K. et al. PennCNV: An integrated hidden Markov model designed for high-resolution copy number variation detection in whole-genome SNP genotyping data. Genome Res. 17, 1665-1674. https://doi.org/10.1101/gr.6861907 (2007).

33. Bouska, A. et al. Genome-wide copy-number analyses reveal genomic abnormalities involved in transformation of follicular lymphoma. Blood 123, 1681-1690. https://doi.org/10.1182/blood-2013-05-500595 (2014).

\section{Acknowledgements}

We wish to thank Mr. Pedram Amouzadeh who assisted in the proof-reading of the manuscript.

\section{Author contributions}

M.Z.A., S.M.N., and A.A. participated in the study design and analysis of the data. M.Z.A. and S.M.N. wrote the manuscript. All authors read and approved the final manuscript.

\section{Competing interests}

The authors declare no competing interests.

\section{Additional information}

Supplementary Information The online version contains supplementary material available at https://doi.org/ 10.1038/s41598-021-00746-0.

Correspondence and requests for materials should be addressed to M.Z.-A.

Reprints and permissions information is available at www.nature.com/reprints.

Publisher's note Springer Nature remains neutral with regard to jurisdictional claims in published maps and institutional affiliations.

(c) Open Access This article is licensed under a Creative Commons Attribution 4.0 International License, which permits use, sharing, adaptation, distribution and reproduction in any medium or format, as long as you give appropriate credit to the original author(s) and the source, provide a link to the Creative Commons licence, and indicate if changes were made. The images or other third party material in this article are included in the article's Creative Commons licence, unless indicated otherwise in a credit line to the material. If material is not included in the article's Creative Commons licence and your intended use is not permitted by statutory regulation or exceeds the permitted use, you will need to obtain permission directly from the copyright holder. To view a copy of this licence, visit http://creativecommons.org/licenses/by/4.0/.

(C) The Author(s) 2021 